\title{
The transport system of nacre components through the surface membrane of gastropods
}

\author{
Elena Macías-Sánchez ${ }^{1, a}$, Antonio G. Checa ${ }^{1, b *}$ and Marc G. Willinger ${ }^{2, c}$ \\ ${ }^{1}$ Department of Stratigraphy and Palaeontology, Faculty of Sciences, Granada, Spain \\ ${ }^{2}$ Electron Microscopy Group, Department of Inorganic Chemistry, Fritz Haber Institute, Max-Planck \\ Society, Berlin, Germany \\ aemacias@ugr.es, ’acheca@ugr.es, cwillinger@fhi-berlin.mpg.de
}

* Corresponding author

Keywords: biomineralization, molluscs, vesicles, calcium

\begin{abstract}
The surface membrane is a lamellar structure exclusive of gastropods that is formed during the shell secretion. It protects the surface of the growing nacre and it is located between the mantle epithelium and the mineralization compartment. At the mantle side of the surface membrane numerous vesicles provide material, and at the nacre side, the interlamellar membranes detach from the whole structure. Components of nacre (glycoproteins, polysaccharides and calcium carbonate) cross the structure to reach the mineralization compartment, but the mechanism by which this occurs is still unknown. In this paper we have investigated the ultrastructure of the surface membrane and the associated vesicle layer by means of Transmission Electron Microscopy. Electron Energy Loss Spectroscopy and Energy-dispersive X-ray Spectroscopy were used for elemental analysis. The analyses revealed the concentration of calcium in the studied structures: vesicles, surface membrane, and interlamellar membranes. We discuss the possible linkage of calcium to the organic matrix.
\end{abstract}

\section{Introduction}

Nacre is a biomineral with a characteristic "brick and mortar" arrangement, which forms the internal layers of the shells of many molluscs. It is produced by the mantle, the epithelium which lines the shell internally. The mantle epithelium secretes parallel membranes (the interlamellar membranes, ILMs) which are mainly composed of glycoproteins and polysaccharides [1,2] between which the aragonite crystals grow. Constituting only $5 \%$ of the dry weight, the organic material confers a resistance to fracture significantly superior to its inorganic counterpart [3]. Bevelander \& Nakahara $[4,5]$ were the first to highlight the importance of these organic sheets in the formation of the molluscan structures.

In bivalves, ILMs are secreted in a confined liquid-filled space $(\sim 100-200 \mathrm{~nm})$, known as the extrapallial space, and nacre grows in a step-like manner $[4,6]$. However, in gastropods nacre tablets are stacked in towers [7], forming pyramids. In the seventies, the acceptance of the hypothesis for the previous formation of lamellar sheets was problematic [8,9] mostly due to the possible disturbance of the organic structure caused by mantle movement. With the aid of Transmission Electron Microscopy (TEM) it was possible to observe the wall-like organic structures [10]. The ILMs derive from a dense surface sheet (the surface membrane, SM) that covers the surface of the nacre mineralization compartment [10]. The side of the SM adjacent to the mantle epithelium is 
composed of a dense organic material at its outermost area and by a compressed arrangement of membranes at the innermost region [10]. The SM may provide a resistant wall to disturbances caused by mantle movement $[11,12]$. At the nacre side of the SM, fresh ILMs progressively detach from it [12,13] and position with a typical spacing of about $500 \mathrm{~nm}$ [6]. ILMs separate progressively from the SM in a zipper-like manner [13].

Since the first description by Nakahara, the SM passed unnoticed until Cartwright \& Checa [13] realized that it is widespread in nacre-secreting gastropods. Checa et al. [12] highlighted the existence of vesicles adhered to the SM at the mantle side. They are round bodies with different morphologies, from spherical to strongly compressed, which seem to fuse with the SM. Their walls are electrodense and have a mean thickness of 10-15 nm. In this way, the SM seems to be formed by the addition of organic vesicles to its mantle-side surface, which gradually integrate into the whole structure.

The SM maintains a constant thickness due to the strict balance between components acquired via vesicle addition and those lost due to the formation of the ILMs [12]. Therefore, it is in a dynamic equilibrium. At the same time, with the incorporation of material and its concomitant loss, the SM moves in an adapertural direction, by keeping pace with the animal's growth rate.

We have studied the SM and its vesicle layer in Phorcus turbinatus (Trochidae, Vetigastropoda). The growth of the nacreous layer takes place close to the margin at the shell aperture [14,15]. It is preceded by a fibrous prismatic layer and followed and covered internally by an aragonitic structure of nacreous aspect (Fig. 1).

We hypothesize that vesicles act as a transport system of the components of nacre to the biomineralization compartment. Our aim is to identify the constituents of those vesicles (proteins, chitin, calcium carbonate, etc.) and characterize, as far as possible, the form or phase in which they travel (monomers or polymers in the case of chitin, and the type of polymorph/polyamorph/ions in the case of calcium carbonate).

\section{Methods}

\section{Sample preparation}

Different preparative protocols were used. Glutaraldehyde fixation followed by osmium tetroxide provides an excellent quality of tissue preservation for the observation of the ultrastructure under the TEM [16]. Nevertheless, it is carried out in an aqueous media, which might wash out the ion content of the sample. Consequently we avoided it for elemental analysis. Testing the content of soft elements in subcellular compartments is challenging due to the difficulty in maintaining the original element distribution. Important advances have been made in cryo-microscopy in the last decade, mostly focusing on cell cultures and single particles. Techniques used here are still in the experimental stages, since they result from adaptations of cryo-techniques not normally used on mineralized samples.

\section{Chemical fixation}

Specimens of Phorcus turbinatus were collected in Benalmádena (Málaga, SE Spain). Immediately after capture, they were fixed in cacodylate-buffered glutaraldehyde $2.5 \%$. Fragments of the SM area were fractured under the binocular microscope, postfixed in osmium tetroxide, and embedded in embedding resin EMbed 812 (EMS). 
Quick-freeze and freeze-drying

Juvenile specimens of Phorcus turbinatus were collected in La Herradura (Granada, SE Spain). The specimens were frozen in liquid nitrogen following collection and maintained in $\mathrm{N}_{2}$ until they were freeze-dried. Quick-freezing immobilizes all molecules in a cell within milliseconds. It is possible to achieve up to $2-20 \mu \mathrm{m}$ of good freezing depth. Beyond that, ice crystals form and destroy the tissue. For freeze-drying, the initial temperature $\left(-170^{\circ} \mathrm{C}\right)$ was raised slowly at an average pressure of 75 millitorr (Flexi-Dry MP) and at the end of the drying cycle (2 days), when room temperature was reached, the specimens were removed and stored at $4^{\circ} \mathrm{C}$.

Fragments from the SM area were cut under the binocular microscope, postfixed in 1\% osmium tetroxide at $4^{\circ} \mathrm{C}$, dehydrated through a series of ethanol solutions and embedded in epoxy resin (EMbed 812). With the aim of comparing the effect of the different fixatives, another set of samples was prepared by avoiding osmium tetroxide postfixation.

Slices were cut (PowerTome Ultramicrotome) at a small angle relative to the surface (less than $10^{\circ}$, Fig. 1 right), thus trying to maximize the area of the cut through the vesicle layer and the SM. The slices were laid in copper grids with lazy carbon to stabilize them under the electron beam. The observations were made at $200 \mathrm{kV}$ in a Philips CM200 and at $300 \mathrm{kV}$ in a FEI Titan.

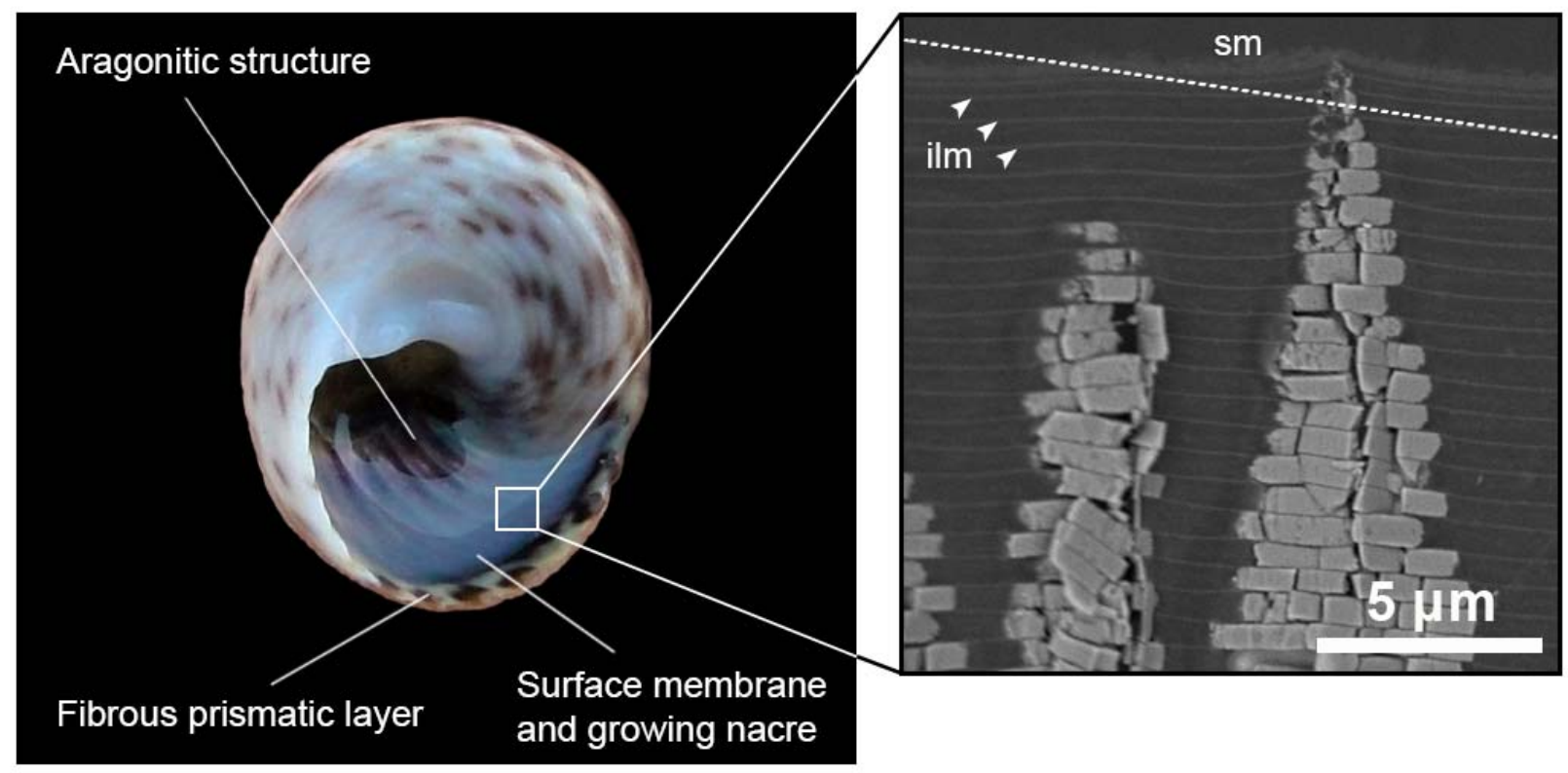

Fig. 1. Left, specimen of Phorcus turbinatus indicating the location of the surface membrane and the growing nacre (sampled area) in relation to the other microstructures present at the shell aperture. Right, SEM image of an ultramicrotome slice perpendicular to the shell surface, where it is possible to distinguish the SM (sm), the interlamellar membranes (ilm) and two towers of growing nacre. The dotted line indicates the approximate angle of the ultramicrotome cuts carried out in this study.

\section{Elemental analysis}

Once the vesicle layer was localized under the TEM, our aim was to know if there was calcium associated with these vesicles. We used two elemental analysis techniques alternatively; Electron Energy Loss Spectroscopy (EELS) and Energy-dispersive X-ray Spectroscopy (EDS). The former 
measures the energy lost by the beam electrons when they interact with the sample, which corresponds with the energy needed to remove one electron from the inner-shell of a particular atom. This is characteristic of each element, and allows us to identify it [17]. EELS is a very powerful technique, since it can detect concentrations of an element greater or equal to $1 \%$. It is highly sensitive to relatively low atomic number elements, where the edges are clear and the energy loss is easy to distinguish. Spectral energy resolution in EELS is much higher than in EDS (about 1 $\mathrm{eV}$ in EELS as opposed to about $125-150 \mathrm{eV}$ in EDS). But what makes EELS an extraordinary useful tool is its capacity to assess atomic composition, chemical bonding and valence state.

EELS spectra were obtained using a post column energy filter spectrometer (GIF Quantum Energy Filter, Gatan) at $200 \mathrm{kV}$ electron accelerating voltage and collection semi-angle of $20.0 \mathrm{mrad}$ with dispersion rate of $0.2 \mathrm{eV} /$ channel. Samples were placed in a single tilt holder and the aperture size on the spectrometer used for data acquisition was $2 \mathrm{~mm}$ in diameter. The acquisition time was optimized to obtain minimum noise and not to provoke beam damage.

EDS measures the number and energy of the X-rays produced by the ionization of the elements in the sample and these X-rays are characteristic of the element and the type of electron transition. EDS has limitations for determining light elements $(Z<4)$, but there is some degree of overlap with respect to atoms such as $\mathrm{Na}, \mathrm{Mg}, \mathrm{P}, \mathrm{S}, \mathrm{Cl}, \mathrm{K}$ and $\mathrm{Ca}(4<\mathrm{Z}<11)$, which can also be detected with EELS. This investigation used an EDS system (EDAX Genesis-4000) coupled with the TEM Philips CM200.

\section{Results}

\section{Ultrastructure}

Samples fixed with glutaraldehyde and postfixed with osmium offered enough contrast to distinguish the vesicle layer under bright field imaging mode (no post stain was used) (Fig. 2a, b), although High-Angle Annular Dark Field (HAADF) - STEM mode offered the best quality (Fig. 3a, $b, e)$. Samples without osmium postfixation did not show enough contrast to detect the vesicles.

The vesicle monolayer contains mostly round vesicles, with an average size $\sim 200 \mathrm{~nm}$ (from 50 to $400 \mathrm{~nm}$ ) (Fig. 2c). A double membrane (10-15 nm) was easily distinguishable (Fig. 2d). Electrodense material seems to fill the interior of the vesicles, attached to the inner membrane (Fig. 2c; Fig. 3b, e).

The SM ( $\sim 100 \mathrm{~nm}$ width) appears like a progressively ordered structure. It has a dense homogeneous structure at the mantle side that organizes progressively into a fibrous arrangement at the nacre side. Towards the interior, the most incipient crystals of the towers appear, and the ILMs seem to detach from the SM (Fig. 3b). 

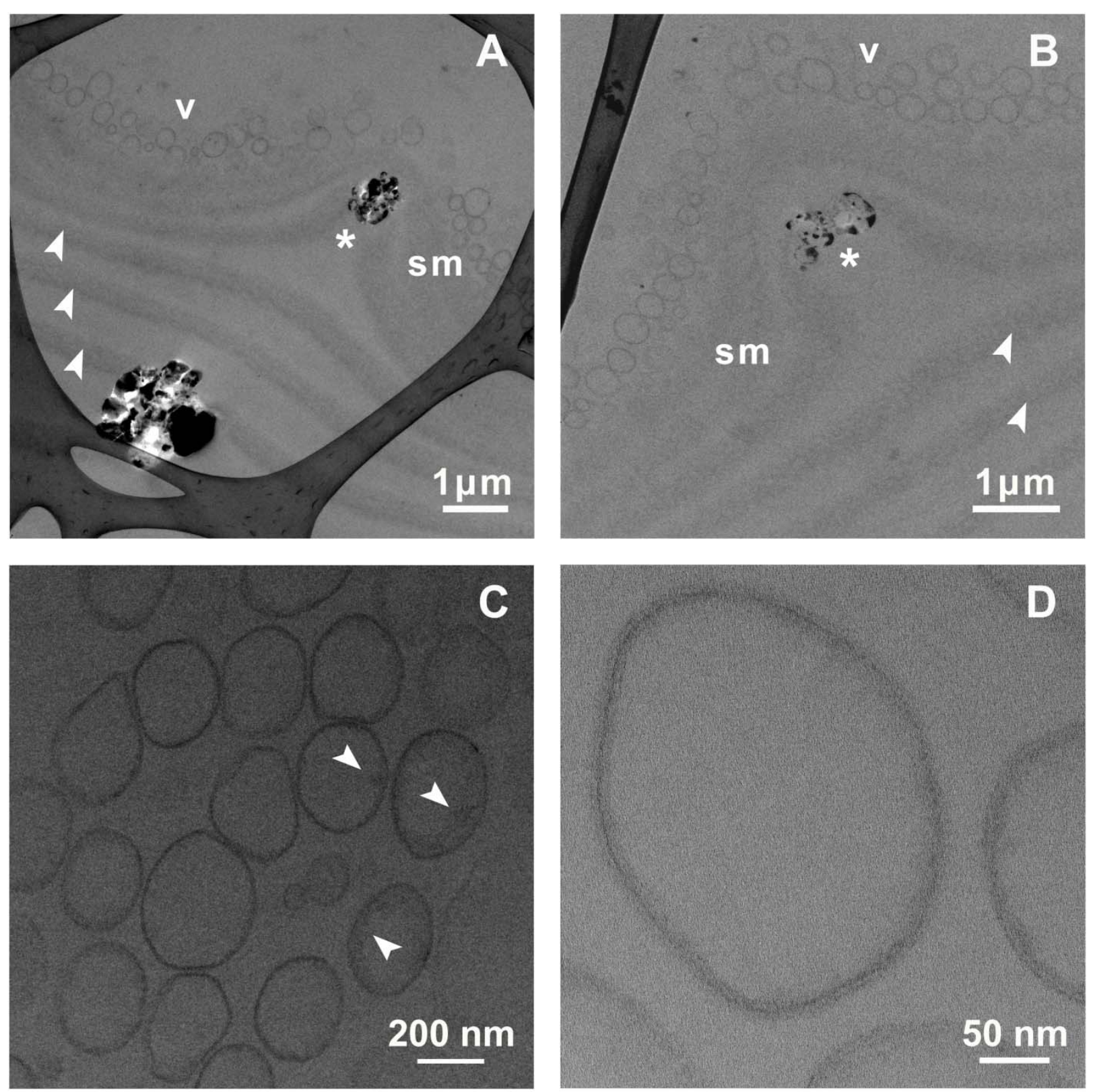

Fig. 2. Bright field TEM images of the vesicle layer. A) and B) vesicle layer (v) covering the surface membrane (sm). The tips of the first towers (marked with asterisks) are partly immersed within the SM (sm), and the interlamellar membranes (arrowheads) detach from it. C) Details of the vesicles showing the material attached to the inner membrane (arrowheads) and D) the double membrane. Samples fixed with glutaraldehyde $2.5 \%$ and postfixed with $1 \%$ osmium tetroxide.

\section{Elemental analysis}

The samples prepared by quick-freeze and freeze-drying preserved the cell structures relatively well. It was possible to localize the areas of interest (SM and vesicle layer), although some movements of the sub-cellular components might have occurred. The maintenance of the ion content was expected to be relatively good, since the sample was in contact with aqueous media only during the dehydration process, after the osmium postfixation. Microanalysis was carried out on the thinnest sections, selected on the basis of their grey hue ( $\sim 50 \mathrm{~nm}$ thickness). 
Systematic EELS measurements were performed on quick-frozen and freeze-dried samples, with and without $\mathrm{OsO}_{4}$ as post-fixative. Signal intensity was higher in samples where osmium tetroxide was used. EDS analysis corroborated the data obtained by EELS. The structures studied in the outer side of the mineralization compartment were: the interior and the membrane of the vesicles ( $\mathrm{v}$ and mv respectively), the SM (sm) and the light areas (la) in the Epoxy resin. In the inner side of the mineralization compartment, the ILMs (ilm), the electrodense material (em) spread between the ILMs and the light areas (la) in the Epoxy resin were analysed. Similar spectra were recorded at each type of the structure investigated, so we present here (Fig. 3h; Fig. 4b) a representative single raw spectrum of each one. No smoothness was applied.

At the outer side of the mineralization compartment, EELS measurements revealed strong carbon $\mathrm{K}$ edges at $285 \mathrm{eV}$, indicating poorly ordered carbon, owing to the Epoxy resin used. The nitrogen $\mathrm{K}$ edge at $400 \mathrm{eV}$ and oxygen $\mathrm{K}$ edge at $532 \mathrm{eV}$ were evident in all the measurements. A strong $\mathrm{Ca}$ $\mathrm{L}_{2,3}$ edge $(\sim 348 \mathrm{eV})$ was detected in the $\mathrm{SM}(\mathrm{sm})$ and in the interior of the vesicles $(\mathrm{v})$. The membrane of the vesicles reported a minor intensity of the $\mathrm{Ca}_{2,3}$ edge and undetectable calcium signal was obtained from the light outer areas (la) (Fig. 3g, h).
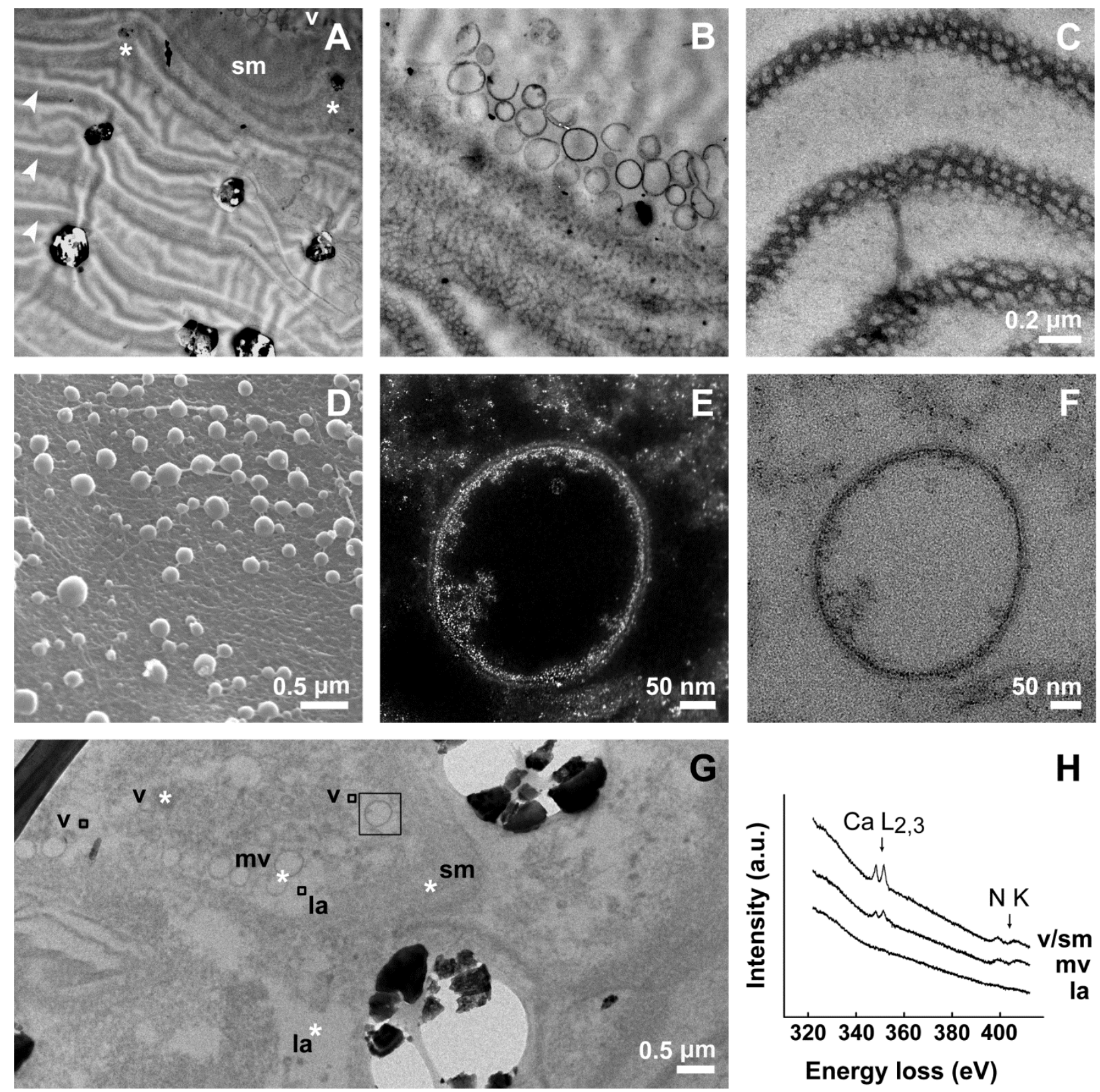
Fig. 3. Surface membrane and vesicle layer of Phorcus turbinatus. A) General view in STEM mode including the vesicles $(\mathrm{v})$, the surface membrane $(\mathrm{sm})$, the first towers immersed in the surface membrane (asterisks) and the interlamellar membranes (arrowheads). B) HAADF image of the vesicle layer, the surface membrane and the interlamellar membranes detaching from it. C) Bright field imaging mode of mature interlamellar membranes with the porous aspect typical of gastropods. Section post-stained with lead citrate and uranyl acetate. D) Scanning electron microscopy image of the vesicles covering the exterior of the surface membrane. E) HAADF image of a vesicle showing the material attached to its inner membrane. F) Same vesicle as in E) but in bright field mode. G) Bright field image of the vesicle layer and the surface membrane in an area close to the nacre towers, showing the vesicles (v), the membrane of the vesicles (mv), the surface membrane (sm) and the light areas (la) of the Epoxy resin. One spectrum was recorded in each point labelled. The vesicle shown in E) and F) is marked with a square. H) Core-loss spectra from the areas marked with an asterisk in $\mathrm{G}$ ), showing $\mathrm{Ca}_{2,3}$ and $\mathrm{N} \mathrm{K}$ edges. Single raw spectra, no smoothness was applied. Intensity is expressed in arbitrary units (a.u.) for comparison. A) and B) samples fixed with glutaraldehyde $2.5 \%$ and postfixed with $1 \%$ osmium tetroxide. C), E), F), G) samples quick-frozen and freeze-dried. D) Critical point dried sample.

At the inner side of the mineralization compartment, EELS spectra from the ILMs (ilm) revealed the $\mathrm{K}$ edges of carbon $(285 \mathrm{eV})$, nitrogen $(400 \mathrm{eV})$ and oxygen $(532 \mathrm{eV})$, as well as $\mathrm{L}_{2,3}$ edge of calcium $(348 \mathrm{eV})$. The spectra obtained from the electrodense material (em) spread in the spaces between the ILMs showed the same peaks, though less intense. In the lighter areas (la) between ILMs, we observed the $\mathrm{K}$ edges of carbon, nitrogen and oxygen that correspond to the embedding resin, and a negligible signal of $\mathrm{Ca}_{2,3}$ edge.
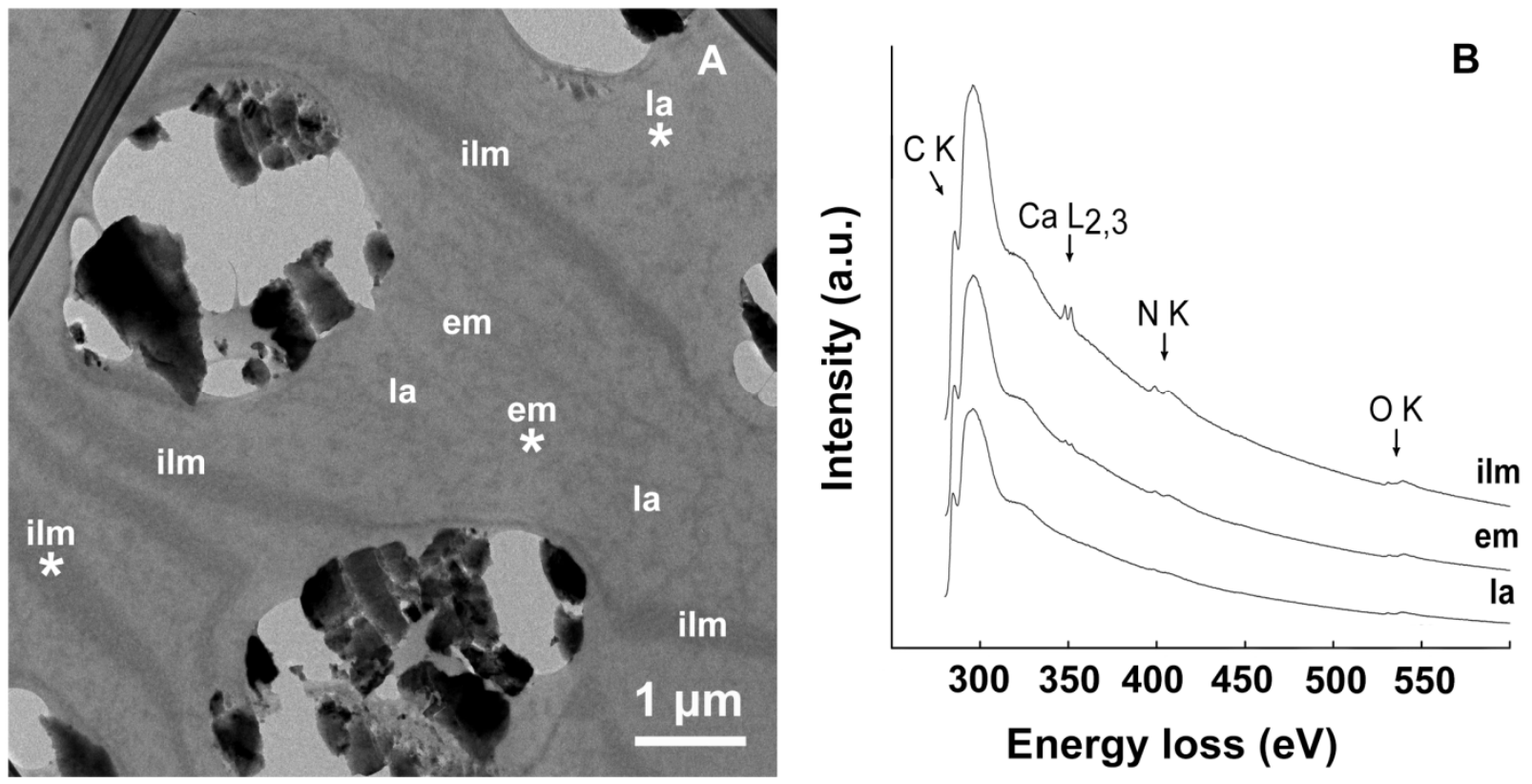

Fig. 4. A) TEM image of the mineralization compartment of a resin embedded section. It is possible to discern the towers of platelets (in diagonal section), the interlamellar membranes (ilm), the electrodense material (em) in between and the light areas (la). One spectrum was recorded in each point labelled. B) Core loss spectra recorded at $200 \mathrm{keV}$ from the areas marked with an asterisk in A (average pixel area $105 \mathrm{~nm}^{2}$ ), showing $\mathrm{C} \mathrm{K}, \mathrm{Ca} \mathrm{L}_{2,3}, \mathrm{~N} \mathrm{~K}$ and O K edges. Single raw spectra, no 
smoothness was applied. Intensity is expressed in arbitrary units (a.u.) for comparison. Quickfrozen and freeze-dried sample, postfixed in osmium tetroxide and embedded in EMbed 812.

\section{Discussion}

Images presented here clearly show double-layered vesicles (Fig. 2d), probably constituted of phospholipids, containing electrodense material. Some of them were filled completely, although others seemed to be partially empty, with the material mostly attached to the inner membrane (Fig. 2c; Fig. 3e, f). The dense amorphous material surrounding the vesicles and covering the SM (Fig. $3 \mathrm{~b}, \mathrm{e})$ might have been extruded from the vesicles in areas adjacent to the SM.

In cross section, the material that makes up the SM seems to undergo maturation and becomes increasingly better defined and organized into layers with depth (Fig. 3b). At the mantle side, it has a homogeneous soft aspect and, beyond this, fibrillar structures separated by irregular holes become perceivable. The typical porous aspect of gastropod ILMs (Fig. 3c) is fully achieved on the nacre side and in some parts it was possible to see an ILM detaching from the SM.

Elemental analysis revealed the presence of calcium in different areas of the system: the dense material inside and surrounding the vesicles, the SM, the ILMs and the electrodense matrix scattered between the ILMs. Two facts suggest that calcium is in a bound form: 1) no calcium was detected in the chemically fixed samples, since chemical fixation and standard preparation protocols involve several aqueous steps in which ions and small molecules could be mobilized. 2) The observation that a strong calcium signal was repeatedly detected in the electrodense material, in contrast to an undetectable signal in the lighter areas of the sample. Calcium has a small $\mathrm{Z}$ number, so it does not give high contrast in bright field imaging mode. The electrodense material with which it seems to be associated is stained with osmium tetroxide that preferentially binds to double bonds of lipids and some proteins.

It is likely that calcium forms part of the structures (SM, ILMs) bound to the organic matrix. It is well established that ILMs are composed mainly of proteins and polysaccharides [18]. SM composition is not known as yet, but it is easy to speculate that it should be similar to that of the ILMs, since these detach from the SM. Similarly, calcium seems to be in a bound form inside the vesicles. The fact that a significantly stronger calcium signal was retrieved from post fixed samples, suggests that fixation with glutaraldehyde followed by $\mathrm{OsO}_{4}$ could avoid the loss of part of the material.

We have to keep in mind that forming nacre tablets begin to grow within the SM [12]. They first acquire their full height and subsequently expand laterally; in this way, the tip of the tower is always embedded within the SM [12]. Previous work has highlighted that the nuclei of gastropods tablets are enriched with organic material $[11,15]$. Accordingly, the organic material that composes the central core of the plates should be absorbed from the SM. In other groups, (Atrina, Bivalvia; Nautilus, Cephalopoda), the core mainly consists of acidic proteins [19] which seem to be distributed concentrically around the nucleus [20].

Recent findings re-emphasize the important role of negatively charged groups in sequestering free calcium ions and promoting nucleation events [21]. Our findings concerning the concentration of the calcium associated with an organic matrix (SM, ILMs) concur with those of Smeets et al. [21]. ILMs are mainly composed of acidic glycoproteins enriched in aspartic and glutamic acid [22], which are negatively charged at cellular $\mathrm{pH}(\mathrm{pH}$ 7) [23]. The calcium atoms of the SM and ILMs might be bound to the negative groups of the matrix. Future efforts should be directed towards 
determining the nature of the content of the vesicles and the mechanisms by which calcium binds to these molecules.

\section{Acknowledges}

We acknowledge Achim Klein-Hoffmann (Fritz-Haber-Institute) for guidance in sample preparation and Xing Huang (Fritz-Haber-Institute) for his advice in analytical techniques. E.M.-S. received funding from project P10-RNM6433 of the Andalusian Consejería de Innovación Ciencia y Tecnología, as well as from the European COST Action TD0903 (STSM-TD0903-010513032973 and STSM-TD0903-090114-039009). Comments from two anonymous reviewers helped to improve the manuscript.

\section{References}

[1] S. Weiner, W. Traub, X-ray diffraction study of the insoluble organic matrix of mollusk shells, FEBS Lett. 111 (1980) 311-316.

[2] S. Weiner, L. Addadi, Acidic macromolecules of mineralized tissues - the controllers of crystal-formation, Trends Biochem. Sci. 16 (1991) 252-256.

[3] A.P. Jackson, J.F.V. Vincent, R.M. Turner, The mechanical design of nacre, Proc. R. Soc. London Ser B 234 (1984) 415-440.

[4] G. Bevelander, H. Nakahara, An electron microscope study of the formation of the nacreous layer in the shell of certain bivalve molluscs, Calcif. Tissue Res. 3 (1969) 84-92.

[5] G. Bevelander, H. Nakahara, Compartment and envelope formation in the process of biological mineralization, in: M. Omori, N. Watabe (Eds.), Mechanism of Biomineralization in Animals and Plants, Tokai University Press, Tokyo, 1980, pp. 19-27.

[6] H. Nakahara, Nacre formation in bivalve and gastropod molluscs, in: S. Suga and H. Nakahara (Eds.), Mechanisms and Phylogeny of Mineralization in Biological Systems, Springer, Berlin, 1991, pp. 343-350.

[7] S. Wise Jr., W. Hay, Scanning electron microscopy of molluscan shell ultrastructures II. Observations of growth surfaces, Trans. Am. Microsc. Soc. 86 (1968) 419-430.

[8] H.K. Erben, Über die Bildung und das Wachstum von Perlmutt, Biomineralization 4 (1972) 1546.

[9] H.K. Erben, On the structure and growth of the nacreous tablets in gastropods, Biomineralization 7 (1974) 14-27.

[10] H. Nakahara, An electron microscope study of the growing surface of nacre in two gastropod species, Turbo cornutus and Tegula pfeifferi, Venus 3 (1979) 205-211.

[11] H. Nakahara, Calcification of gastropode nacre, in: P. Westbroek, E.W. De Jong (Eds.), Biomineralization and Biological Metal Accumulation, Reidel Publishing Company, Dordrecht, 1983, pp. 225-230. 
[12] A.G. Checa, J.H.E. Cartwright, M.G. Willinger, The key role of the surface membrane in why gastropod nacre grows in towers, Proc. Nat. Acad. Sci. USA 106 (2009) 38-43.

[13] J.H.E. Cartwright, A.G. Checa, The dynamics of nacre self-assembly, J. R. Soc. Interface 4 (2007) 491-504.

[14] S. Wise Jr., Microarchitecture and deposition of gastropod nacre, Science 167 (1970) 14861488.

[15] H. Mutvei, Ultrastructural characteristics of the nacre in some gastropods, Zoologica Scripta 7 (1978) 287-296.

[16] M.A. Hayat, Basic Techniques for Transmission Electron Microscopy, first ed., Academic Press, Orlando, 1986.

[17] R.F. Egerton, Electron Energy-Loss Spectroscopy in the Electron Microscope, third edition, Springer, US, 2011.

[18] G. Goffinet, Ch. Jeuniaux, Composition de la conchioline du nacre de Nautilus, Comp. Biochem Physiol 29 (1969) 277-282.

[19] M.A. Crenshaw, H. Ristedt, Histochemical and structural study of nautiloid septal nacre, Biomineralization 8 (1975) 1-8.

[20] F. Nudelman, B.A. Gotliv, L. Addadi, S. Weiner, Mollusk shell formation: mapping the distribution of organic matrix components underlying a single aragonitic tablet in nacre, J. Struct. Biol. 153 (2006) 176-187.

[21] P.J.M. Smeets, K.R. Cho, R.G.E. Kempen, N.A.J.M. Sommerdijk, J.J. De Yoreo, Calcium carbonate nucleation driven by ion binding in a biomimetic matrix revealed by in situ electron microscopy, Nature Materials (2015) Advance online publication, doi: 10.1038/nmat4193

[22] S. Weiner, Aspartic acid-rich proteins: major components of the soluble organic matrix of mollusk shells, Calcif. Tissue Int. 29 (1979) 163-167.

[23] D.L. Nelson, M.M. Cox, Lehninger Principles of Biochemistry, fourth ed., W.H. Freeman and Company, New York, 2005. 